\title{
Efficacy and compliance of a prehospital spinal immobilization guideline
}

\author{
Lucas A. Myers $\cdot$ Christopher S. Russi • \\ Daniel G. Hankins $\cdot$ Kathleen S. Berns $\cdot$ Scott P. Zietlow
}

Received: 21 November 2008 / Accepted: 1 January 2009/Published online: 14 February 2009

(C) Springer-Verlag London Ltd 2009

\begin{abstract}
Background Prehospital spinal immobilization criteria are useful in identifying those at risk for spinal fractures, while reducing the number of patients unnecessarily immobilized. The use of immobilization criteria, without regard to mechanism of injury, has been shown to accomplish this task. Aims The study's purpose is to examine efficacy of a prehospital spinal clearance guideline and triage/management of these injuries.

Methods This was a retrospective study of traumatically injured patients based on a clinical clearance spinal immobilization guideline between January 2006 and January 2007. Two gold standards were used in the analysis (radiographic findings and physician clearance without radiographs). This
\end{abstract}

The views expressed in this paper are those of the author(s) and not those of the editors, editorial board or publisher.

\section{A. Myers $(\bowtie)$}

Mayo Clinic Medical Transport,

501 6th Ave NW,

Rochester, MN 55901, USA

e-mail: myers.lucas3@mayo.edu

C. S. Russi • D. G. Hankins

Department of Emergency Medicine, Mayo Clinic,

1216 Second Street SW,

Rochester, MN 55902, USA

K. S. Berns

Mayo Clinic Medical Transport,

1216 Second Street SW,

Rochester, MN 55902, USA

\section{S. P. Zietlow}

Division of Trauma, Critical Care and General Surgery,

Mayo Clinic,

1216 Second Street SW,

Rochester, MN 55902, USA project was approved by the Mayo Clinic Institutional Review Board.

Results The study included 942 patients documented to have a traumatic injury. Of these, $43(4.6 \%)$ had an acute spinal fracture. The guideline allowed 558 (59.2\%) patients to be cleared, and 1.3\% (7/558) had fractures. The remaining 384 did not meet clearance criteria and accounted for $36(9.4 \%$, $36 / 384$ ) fractures. The guideline correctly predicted 36 of 43 fractures. The median age of the 7 fractures not immobilized was 82 years and of the 36 patients with fractures that were immobilized was 48 years. When immobilization was indicated, caregivers were $77.6 \%$ (298/384) compliant. Of the noncompliant $22.4 \%(86 / 384)$ there were 9 fractures.

Conclusions This spinal guideline demonstrates efficacy in identifying those at risk for spinal fractures. An age extreme criteria may enhance this already effective guideline. Further analysis of compliance failures may improve the guideline's ability for fracture prediction.

Keywords Prehospital trauma - Immobilization .

Spinal clearance Ambulance - Compliance .

Spinal fracture $\cdot$ Prehospital

\section{Introduction}

Nearly 200,000 Americans live with spinal cord injury (SCI), and approximately 11,000 more sustain SCI annually [1]. The leading causes of injury - motor vehicle collisions, falls, and acts of violence - commonly require an ambulance response. Any of these mechanisms of injury (MOIs) may cause SCI.

It is the responsibility of emergency medical services (EMS) to transport the sick and injured without causing or worsening injuries. Devices for spinal immobilization such as 
the long back board, cervical collar, and Kendrick extrication device are commonly used to prevent movement and further injury in patients suspected of having spinal fractures.

Ambulance medical guidelines exist to determine which patients have the highest potential for SCI and, therefore, need immobilization. Inclusion of the MOI as an indicator of injury has long been part of prehospital medical guidelines. Selective spinal clearance (i.e., no immobilization required) guidelines, without regard for the MOI, may be an effective tool for reducing unnecessary immobilization while accurately identifying spinal fractures [2-5]. These reports also demonstrate selective immobilization criteria to be effective for identifying those at highest risk for cervical spinal injury and reducing the need for hospital radiographs $[6,7]$.

Although the selective immobilization procedure has shown effectiveness, little has been evaluated concerning compliance with guidelines specifically by ambulance caregivers. The selective immobilization guideline allows for greater interpretation and relies heavily on the assessment skills of paramedics and emergency medical technicians (EMTs).

This retrospective cohort study aimed to evaluate the performance of a corporate EMS guideline to recognize spinal fractures and assesses prehospital compliance with the guideline and subsequent patient outcomes.

\section{Methods}

\section{Study design}

This study evaluated all patients identified and documented to have a traumatic injury and transported to Saint Marys Hospital, a level 1 trauma center in Rochester, MN, an urban, Midwestern city in United States. The study examined whether spinal immobilization was correctly performed by prehospital providers and whether a spinal fracture was found at the receiving level 1 trauma center. The study investigators reviewed EMS records spanning 1 year (1 January 2006 through 31 December 2006).

Each patient was screened for spinal immobilization using an institutional spinal immobilization guideline in place since 2002. The guideline allows exclusion of spinal immobilization if all the following criteria are met:

- No pain, stiffness, soreness, or tenderness in the neck or back

- No alteration in level of consciousness

- No intoxication from alcohol or other drugs

- No other painful or distracting condition that may interfere with the patient's perception of spinal injury symptoms

- No signs or symptoms of shock
Patients with suspected traumatic spinal injury were included for analysis on the basis of MOIs, patient complaints, treatments performed, or primary diagnosis in the prehospital medical record. The electronic prehospital record allows for traumatic MOIs to be categorized into five fields: falls, motor vehicle collisions, sports-related injuries, violence, and other. The MOI for each patient was documented. Hospital patient records were also evaluated, specifically emergency department (ED) notes and radiographic tests. Neurologic status at hospital discharge was the study's end point. Two gold standards were used in the analysis (radiographic findings and physician clearance without radiographs). Data were collected and analyzed using JMP software (SAS Institute, Cary, NC, USA). This project was approved by the Mayo Clinic Institutional Review Board.

\section{Patients}

Included were all patients with traumatic MOIs (with the exception of patients undergoing interfacility transport or ambulance intercepts) who were transported to a level 1 trauma center. Also excluded were patients transported to a hospital other than the study hospital as well as those who did not grant research authorization.

\section{Results}

The study identified 942 patients documented to have a traumatic injury. Of these, $43(4.6 \%)$ had acute spinal fractures identified by hospital radiography, computed tomographic (CT) scan, or both. The guideline correctly predicted 36 of 43 patients $(83.7 \%)$ (95\% confidence interval: $86.9-92.9 \%$ ) identified to have spinal fractures.

A total of 384 patients $(40.8 \%)$ met the criteria to be immobilized. Of the patients meeting selective criteria for immobilization, $36(9.4 \%)$ were found to have a spinal fracture. ED radiography for the purpose of spinal evaluation was performed on 323 of the 384 patients ( $84.1 \%$ ). Of the 384 patients meeting immobilization criteria, 298 (77.6\%) were actually immobilized. Hence, noncompliance with the guideline was found in 86 patients $(22.3 \%)$ and accounted for 9 of the 36 acute spinal fractures $(25.0 \%)$ in this group. ED radiography was performed on 64 of the 86 patients (74\%) without prehospital immobilization.

The patient record was evaluated to determine reasons for noncompliance as shown in Table 1. The documentation was reviewed to determine if patients were intoxicated as per the paramedic/EMT narrative. The narrative conclusions for intoxication were not confirmed by hospital laboratory tests. A paramedic's assessment of intoxication required the paramedic to immobilize the patient according to the guideline. 
Table 1 Reasons for noncompliance

\begin{tabular}{llll}
\hline Reason & No. & Films & Fracture \\
\hline $\begin{array}{l}\text { Intoxication } \\
\text { Crew (no clear documentation }\end{array}$ & 29 & 18 & 0 \\
$\quad$ or reasoning) & & 20 & 4 \\
$\begin{array}{l}\text { Disorientation (unresponsiveness } \\
\quad \text { or dementia) }\end{array}$ & 13 & 12 & 1 \\
$\quad \begin{array}{l}\text { One device only (LBB or C collar) } \\
\text { Communication barrier (including }\end{array}$ & 8 & 8 & 3 \\
$\quad$ children <1 year) & 4 & 3 & 0 \\
$\begin{array}{l}\text { Patient refusal } \\
\text { Other distracting injury }\end{array} \quad 4$ & 3 & 1 \\
$\quad$ as defined by MD) & 2 & 1 & 0 \\
\hline
\end{tabular}

$C$ collar cervical collar; $L B B$ long back board

The guideline allowed for the clearance (no immobilization) of 558 patients (59.2\%). ED radiography was obtained on 101 of these patients (18.1\%) and subsequently identified 7 $(6.9 \%)$ to have spinal fractures. Eighty-five patients were immobilized despite the guideline's allowing clearance. Of those 85 patients, 3 (3.5\%) had spinal fractures. Even though the guideline allowed clearance, there was a difference in radiographic films obtained. ED physicians obtained radiography in $14.2 \%$ of patients $(67 / 473)$ who were not immobilized. The 85 patients who were immobilized had a $40 \%$ rate $(34 / 85)$ of radiography acquisition.

\section{Mechanisms of injury}

As shown in Table 2, the five MOIs as documented by ambulance staff are listed as percentages of the total (942) and categorized by immobilization group. Falls accounted for 513 of the total 942 patients (54.4\%) screened with the selective immobilization guideline. As noted in Table 3, falls also accounted for the largest group for which the guideline allowed clearance [335/942 (35.6\%)]. In the 513 patients in the falls group, 21 acute spinal fractures (4.1\%) were diagnosed. This group also accounted for the highest rate of noncompliance with the immobilization guideline. Immobilization was indicated but not performed in 47 of the total $513(9.2 \%)$, including 6 of the 21 (28.6\%) found to have a spinal fracture.

Motor vehicle collisions accounted for 249 of all 942 traumatic injuries $(26.4 \%)$ but had the highest rate of spinal fractures, more than $8 \%(20 / 249)$. Three of those fractures were missed by the guideline $(10 \%)$.

Violence accounted for 126 of all 942 traumatic injuries $(13.4 \%)$. Of those patients, one spinal fracture $(0.8 \%)$ was diagnosed.

Twenty-seven patients $(2.9 \%$ of total) had a sportsrelated injury. One of those patients had a spinal fracture.

An additional 27 patients ( $2.9 \%$ of total) were categorized as "other." These injuries included machinery- and workrelated accidents that did not qualify for categorization in other groups. No spinal injuries were identified in this group.

\section{Spinal injuries and outcomes}

Two patients had lasting paralysis after their injuries, both cervical fractures. Both were identified by the guideline and immobilized by ambulance staff. In all 43 fractures, 8 were cervical, 13 thoracic, and 22 lumbar. Of the seven that were not predicted by the guideline, four were lumbar and three thoracic. None of these seven patients had lasting neurologic deficits or cord injuries secondary to the fractures. Of patients admitted (Table 3), those with fractures that met clearance criteria had a 5.1-day average length of stay, and the 36 patients with predicted fractures had a 6.1-day average length of stay.

\section{Discussion}

The selective spinal immobilization guideline allowed for more than half $(59.2 \%)$ of all traumatic injuries to be

Table 2 The five mechanisms of injury and how the study group fit the clearance guideline ${ }^{\mathrm{a}}$

\begin{tabular}{|c|c|c|c|c|c|c|c|c|c|}
\hline \multicolumn{10}{|l|}{ Per protocol } \\
\hline \multirow[t]{2}{*}{ Mechanism } & \multicolumn{2}{|c|}{$\begin{array}{l}\text { Clearance allowed } \\
\text { per guideline }\end{array}$} & \multicolumn{2}{|c|}{$\begin{array}{l}\text { Clearance allowed } \\
\text { but immobilized }\end{array}$} & \multicolumn{2}{|c|}{$\begin{array}{l}\text { Immobilization indicated } \\
\text { and performed }\end{array}$} & \multicolumn{2}{|c|}{$\begin{array}{l}\text { Immobilization indicated } \\
\text { but not performed }\end{array}$} & \multirow{2}{*}{$\begin{array}{l}\text { Total } \\
\text { No. }\end{array}$} \\
\hline & No. & $\%$ & No. & $\%$ & No. & $\%$ & No. & $\%$ & \\
\hline Falls & 298 & 31.6 & 37 & 3.9 & 130 & 13.8 & 48 & 5.1 & 513 \\
\hline MVC & 52 & 5.5 & 43 & 4.6 & 139 & 14.8 & 15 & 1.6 & 249 \\
\hline Other & 18 & 1.9 & 2 & 0.2 & 6 & 0.6 & 1 & 0.1 & 27 \\
\hline Sports & 17 & 1.8 & 0 & 0.0 & 9 & 1.0 & 1 & 0.1 & 27 \\
\hline Violence & 88 & 9.3 & 3 & 0.3 & 14 & 1.5 & 21 & 2.2 & 126 \\
\hline
\end{tabular}

$M V C$ motor vehicle collision

${ }^{\mathrm{a}}$ The percentages are based on the total number of patients (942) 
Table 3 Patient demographics and trauma mechanisms ${ }^{a}$

\begin{tabular}{lllll}
\hline Characteristic & Immobilized $(n=384)$ & Fractures $(n=36)$ & Did not meet criteria $(n=558)$ & Fractures $(n=7)$ \\
\hline Median age, years & 40.5 & 48 & 55.5 & 82 \\
Male sex & $208(54.2)$ & $19(52.8)$ & $247(44.3)$ & $3(42.9)$ \\
Admitted & $203(52.9)$ & $34(94.4)$ & $235(42.1)$ & $6(85.7)$ \\
Spine radiography & $323(84.1)$ & $36(100)$ & $101(18.1)$ & $7(100)$ \\
Falls & $178(46.3)$ & $17(47.2)$ & $335(60)$ & $4(57)$ \\
MVC & $154(40.1)$ & $17(47.2)$ & $95(17)$ & $3(43)$ \\
Violence & $35(9.1)$ & $1(2.8)$ & $91(16.3)$ & $0(0)$ \\
Sports & $10(2.6)$ & $0(0)$ & $20(3.7)$ & $0(0)$ \\
Other & $7(1.9)$ & & & $0(0)$ \\
\hline
\end{tabular}

$M V C$ motor vehicle collision

${ }^{a}$ Values are number (percentage) unless indicated otherwise

cleared from the use of spinal immobilization devices before their arrival to the ED.

The guideline failed to identify 7 of 43 spinal fractures $(16.3 \%)$. This percentage is slightly higher than reports of other selective spinal immobilization guidelines in which missed fracture rates are between 8 and $13 \%[4,6]$. However, in this study, an additional 9 of the 43 (20.9\%) fractures were identified by the guideline but not immobilized by ambulance personnel. In all, 37.2\% (16/42) of patients identified as having a spinal fracture by hospital radiography were either not immobilized or immobilization was not required by the guideline. The overall noncompliance rate was $22.4 \%$ (86/384). Noncompliance raises concerns for the practical use of the guideline. Alcohol intoxication and nonspecified noncompliance accounted for $64.0 \%(55 / 86)$ of all cases of noncompliance. Selective immobilization guidelines rely heavily on the paramedic's assessment and interpretation. Continuing education and compliance were not measured throughout the period this study examined. Introduction of quality measurements as well as continuous review may improve compliance.

When comparing groups by age (shown in Table 3), those that required immobilization were a median of 15 years older than those allowed for clearance. In analyzing the spinal fractures of patients who met criteria to immobilize and to clear, the findings were more dramatic. The median age in the clearance group was 82 years, compared with the median of 48 years in the immobilization group. The addition of cautionary use in the guideline at extreme ages may provide benefit [3]. The prevalence of osteoporosis in the USA may be a reason to include this precaution. It affects $55 \%$ of people older than 50 years in the USA [7]. Vertebral fractures are the most common type of osteoporotic fracture, with an estimated annual incidence of 700,000 in the USA [8]. The prevalence of this disorder supports its inclusion as immobilization criteria for elderly patients (65 years and older). In this study, the hypothetical inclusion of an age- extreme criterion ( $\geq 64$ years) would have identified six of the seven patients not identified by the current guideline. Thus, the accuracy of identifying those with fractures would have increased from 83.7 (36/43) to $97.7 \%$ (42/43).

The inclusion of an age-specific criterion, however, would increase the number of patients requiring immobilization. By our measures, the addition of an age-specific guideline of 64 years or older would have increased the guideline's effectiveness by 14 percentage points; it would also have increased the number of those requiring immobilization by $41.9 \%$ (234/558). Increasing the age to 80 years would have increased the guideline's effectiveness by 9.3 percentage points (to $93.0 \%$ ), but increasing the immobilization number $24.6 \%$ (137/558).

Review of radiographs acquired by physicians suggests that the ambulance providers' decision to immobilize, regardless of the guideline, may influence the decision of the physician to obtain radiographs. When the paramedics were noncompliant in immobilizing (86 patients), they received 64 radiographs $(74.4 \%)$, a $12.5 \%$ lower likelihood of receiving ED radiographs than the patients who were correctly immobilized. Of the 298 patients that were correctly immobilized, $86.9 \%$ (259/298) had radiographs. Conversely, the patients who did receive immobilization, even while the guideline allowed for clearance (34/85), had a $25.8 \%$ higher rate of ED spinal radiographs, CT scans, or both than those who were not immobilized (67/473).

\section{Limitations}

The limitations of a retrospective study must be acknowledged. Patients in the study were transported to one of two local hospitals. Patient records were reviewed for only those patients transported to the hospital with the level 1 trauma center. There was no follow-up of patients who did not undergo radiography in the ED, and their missed injury was not represented in this study. 


\section{Conclusion}

This spinal immobilization guideline demonstrates efficacy in identifying those at risk for spinal fractures. The guideline accurately identified all cervical fractures found in this study. The use of an age-extreme criterion may enhance this already effective guideline. Further analysis of compliance failures may add to the guideline's ability to predict fractures.

More than 20\% (9/42) of patients who had spinal fractures found in this study had indications for immobilization, but it was not performed by ambulance staff. Continual training and regular case review with quality assurance programs should frequently evaluate spinal clearance guidelines. Quality assurance, patient follow-up, and audit systems may improve compliance. It is imperative that ambulance systems monitor and continually review this guideline and train for its application.

Acknowledgments Thanks to Daniel Roline for his guidance and mentorship.

\section{Conflicts of interest None.}

\section{References}

1. Centers for Disease Control and Prevention (1998) http://www.cdc. gov/ncipc/factsheets/scifacts.htm
2. Domeier RM, Evans RW, Swor RA, Hancock JB, Fales W, Krohmer J, Frederiksen SM, Shork MA (1999) The reliability of prehospital clinical evaluation for potential spinal injury is not affected by the mechanism of injury. Prehosp Emerg Care 3 (4):332-337

3. Hoffman JR, Mower WR, Wolfson AB, Knox TH, Zucker MI (2000) Validity of a set of clinical criteria to rule out injury to the cervical spine in patients with blunt trauma. National Emergency X-Radiography Utilization Study Group. N Engl J Med 343(2): 94-99

4. Stroh G, Braude D (2001) Can an out-of-hospital cervical spine clearance protocol identify all patients with injuries? An argument for selective immobilization. Ann Emerg Med 37(6):609-615

5. Domeier RM, Frederiksen SM, Welch K (2005) Prospective performance assessment of an out-of-hospital protocol for selective spine immobilization using clinical spine clearance criteria. Ann Emerg Med 46(2):123-131

6. Burton JH, Dunn MG, Harmon NR, Hermanson TA, Bradshaw JR (2006) A statewide, prehospital emergency medical service selective patient spine immobilization protocol. J Trauma 61 (1):161-167

7. National Osteoporosis Foundation http://www.nof.org/osteoporosis/ diseasefacts.htm

8. Riggs BL, Melton LJ 3rd (1995) The worldwide problem of osteoporosis: insights afforded by epidemiology. Bone 17(5 Suppl):505S-511S

Lucas Myers is a research coordinator and paramedic for Mayo Clinic Medical Transport/Gold Cross Ambulance in Rochester, MN. 